\title{
Awake Non-entubated Video Assisted Thoracoscopic Surgery
}

\author{
Fazli Yanik* \\ Department of Thoracic Surgery, Trakya University, Turkey
}

Submission: August 03, 2017; Published: December 12, 2017

*Corresponding author: Fazli Yanik, Department of Thoracic Surgery, Trakya University, Turkey, Email: fazliyanik@hotmail.com

\begin{abstract}
Abstact
Thoracic surgery developed in greater extent with equipment sand techniques in one lung ventilation in the last decades. Traditionally, VATS is performed under general anesthesia with selective ventilation with double lumenendo trachealintubation. Awake video-assisted thoracic surgery (AVATS) has been increasingly employed in a variety of procedures involving pleura, lungs, and mediastinum. Inthoracic surgery intubation and techniques without in tubation leads different physiologic changes. Adequate anesthesia and analgesia obtained from Thoracic Epidural Anesthesia (TEA), thoracic paravertebral anesthesia(TPA), localanesthesia, intercostal nerve blocks with / without sedoanalgesia allow VATS to be performed in awake patients. AVATS has been demonstrated to be a safe option for thoracic procedures with results rivalling and often exceeding thoracotomy or traditional VATS. The procedure can have such advantages as early mobilization, opening of early oral intake, early discharge, patient satisfaction, low pain level. With careful patient selection, detailed preoperative planning and calm, meticulous operative approach allows using the AVATS technique in even more complex thoracic cases. Nevertheless, there is a need for randomized controlled trials involving wider case series on the subject.
\end{abstract}

Keywords: Thoracic Surgery, Vats, Awake, Non-Entubated

\section{Introduction}

Thoracic surgery developed in greater extent with equipments and techniques in one lung ventilation in the last decades. Still general anesthesia in one lung ventilation approved as gold standard. The reasons for preference of awake patient includes; avoidance of several complications related to general anesthesia. Inthoracic surgery most performed surgeries are plerural decortication and lung biopsy. Avoidance of intubation in Video Assisted Thoracoscopic Surgery (VATS) procedures gains us some advantages in post operative period; a better respiratory outcome, survival and morbidity mortality rates, reduce hospitalization time and costs, reduced early stress hormone and immune response. Traditionally, VATS is performed under general anesthesia with selective ventilation with double lumen endotracheal intubation. Awake video-assisted thoracic surgery (AVATS) has been increasingly employed in a variety of procedures involving pleura, lungs, and mediastinum. Inthoracic surgery intubation and techniques without intubation leads different physiologic changes. With intubation diphragm is completely curarized and mechanical ventilation takes in action. However in nonintubated techniques patients' breathe spontanously and diaphragm muscle contracts
Awake VATS operations without mechanical ventilation were earlier used only for pleural biopsy for diagnostic purposes. However, by the advances in minimally invasive technology such as spontaneous pneumothorax surgery, bullous emphysema, pulmonary nodule resection of lung volume reduction surgery, the decortication, and treatment of mediastinal mass and in lung anatomic resection, in recent year simplementation of case series and randomized controlled trials are available showing that AVATS is a safe and effective technique. Adequate anesthesia and analgesia obtained from Thoracic Epidural Anesthesia (TEA), thoracic paravertebral anesthesia (TPA), local anesthesia, intercostal nerve blocks with /without sedoanalgesia allow VATS to be performed in awake patients. The potential general anesthesia-related adverse effects, such as intubationrelatedtrauma, pneumonia, ventilator-associated lunginjury, effects of neuro muscular blocking agents, and post operative nausea and vomiting, can thus be avoided. Potential cases for AVATS technique performed with awake regional anesthesia are minor cases for healthy patients and high-risk cases where intubation is inconvenienttoapply. The short duration of the operation and careful patient selection is necessary. 


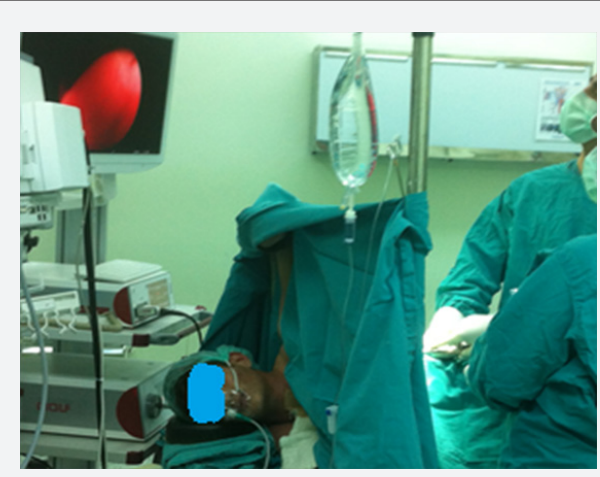

Figure 1: Intraoperative image of the patient who under went AVATS for spontaneous pneumothorax in our clinic.

The unwillingness of the patient to be awaked uring operation, a risk of contamination of the other lung, massive bleeding, infectious conditions such as inflammation, alveolar proteinosis, bronchopleural fistula is among the contra indications of the procedure. In some demanding procedures surgeons prefer thoracicepidural anesthesia which grants superior thoracic analgesia. Morover hypoxemia and hyper capnia may still exist in nonin tubated thoracic surgery. In later aldecubitus position AVATS preserves ventilation perfusion match by ventilation of dependent he midiaphragm in comparison to intubated one-lung ventilationunder general anesthesia. Usually mildhy percapniamay ocur which is well-tolerated and when patient starts two-lung breathing carbondioxide levels return to normal. Additional oxygen therapy by a face mask usually sufficient after surgery. The rates of general anesthesia and intubation are between $2.7 \%$ and $4.3 \%$, depending on the surgical procedure and learning curve applied. Some current articles reported that in $\mathrm{n}$ case of awake technique, patient satisfaction is more, pain levels are less,nursing care is less, the length of hospital stay is shorter and it is safe and feasible surgery. Early discharge, early mobilization and early oral intake is possible with this procedure.

Those patients whose AVATS is contraindicated: Hemodynamically unstable patients, expected difficult airway management (Mallampati III-IV), obesity (BMI>30), intensive pleuraladhesions, inexperienced surgical team in VATS, paradiaphragmaticlesions, large / central pulmonarylesions ( $>6$ $\mathrm{cm}$ ), obstacles to the application of thoracicparavertebralepiuralanesthesia, psychological aspectsareunstable. AVATS has been demonstrated to be a safe option for thoracic procedures with results rivalling and often exceeding thoracoto my ortraditional VATS. Pleural procedures, wed geresections and lobectomies have been perfor medvia AVATS with good results but there has been a paucity of reports regarding AVATS for more complex procedures. Reported mortality rates in patients with malignancy orpoorcardio pulmonary status are often shown to be highest and these patients may benefit most from the AVATS approach. Inconclusion AVATS procedure sare feasibile and safety with minimal adverseevents in the appropriate patient group. The procedure can have such advantages as early mobilization, opening of early oral intake, early discharge, patient satisfaction, low pain level. With careful patient selection, detailed preoperative planning and calm, meticulous operative approach allows using the AVATS technique in even more complex thoraciccases. Never the less, there is a need for randomized controlled trials involving wider casese ries on the subject.

\section{References}

1. Hung MH, Hsu HH, Cheng YJ, Shing J (2014) Nonintubated thoracoscopic surgery: state of the art and future directions. J Thorac Dis 6(1): 20-29.

2. Rocco G (2016) Non-intubated uniportal lung surgery. Eur J Cardio thorac Surg 49(1): i3-i5.

3. Kao MC, Lan CH, Huang CJ (2012) Anesthesia for Awake video assisted thoracic surgery. Acta Anaesthesiol Taiwan 50(3): 126-130.

4. Pompeo E, Mineo D, Rogliani P, Sabato AF, Mineo TC (2004) Feasibility and results of awake thoracoscopicre section of solitary pulmonary nodules. Ann Thorac Surg 78(5): 1761-1768.

5. Chen KC, Cheng YJ, Hung MH, Tseng YD, Chen JS (2012) Non intubated thoracoscopiclung resection: a 3-year experience with 285 cases in a single institution. J Thorac Dis 4(4): 347-351.
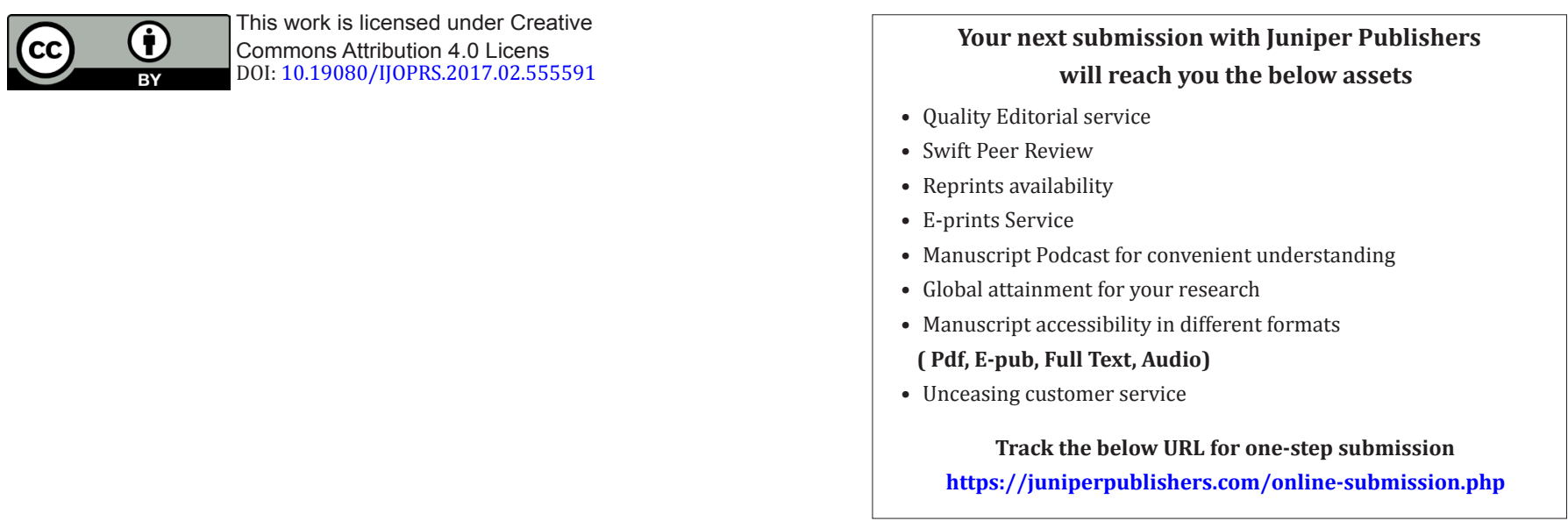